Highly Energetic Physical Processes and

Mechanisms for Emission from Astrophysical Plasmas

IAU Symposium, Vol. 195, 2000

P. C. H. Martens, S. Tsuruta, and M. A. Weber, eds.

\title{
Recent Observations of X-ray Emission from Galactic Binary Neutron Stars
}

\author{
J. Swank \\ NASA/Goddard Space Flight Center, Code 662, Greenbelt, MD 20771, \\ U.S.A.
}

\begin{abstract}
Recent measurements of young, accreting binary neutron stars are determining more precise magnetic field and accretion parameters. A low magnetic field, accreting, millisecond pulsar has finally been found in a binary burster. At least 20 low-mass binaries have exhibited high frequency oscillations, 300-1200 hertz. The majority have, for some range of luminosity, the pair of quasiperiodic oscillations that have never been seen in a black hole candidate. Recent evidence from burst oscillations strengthens the case that the difference frequency of this pair is close to the spin frequency of the neutron star. These oscillations are correlated with the spectra, luminosity, and low frequency oscillations. Quasiperiodic oscillations are also seen sometimes in strong magnetic field pulsars, where their origin can be closely examined.
\end{abstract}

\section{Introduction}

It is now believed that we have galactic, binary neutron stars with magnetic fields that are as low as $10^{8}$ Gauss and as high as $5 \times 10^{14}$ Gauss. There are single neutron stars with magnetic fields as low and probably single neutron stars with a field as high. Because of recent measurements, it is now possible to plot the magnetic fields and rotation periods of representative members of the varied populations. These are displayed in Figure 1, along with the fields and periods of some related objects. The evolutions of systems from birth of the neutron stars to their place in this diagram are subjects in themselves. Only now are the X-ray measurements establishing the places of the objects with reasonable accuracy rather than as an order of magnitude hypothesis.

There are many aspects of the accretion onto the binary neutron stars that are not yet understood. I will touch upon some of these. But observations by RXTE and BeppoSAX have established some facts that vindicate former years of assumptions, enable calculations to be done that depend on the parameters that were determined, and lead to a new round of questions.

\section{Pulsars}

\subsection{Cyclotron Resonance Features in High Magnetic Field Pulsars}

The "classic" accreting binary pulsars are those that were first found in X-ray astronomy with rocket flights and UHURU. Her X-1 with its $1.24 \mathrm{~s}$ period and 
$X$-RAY BINARY PULSARS \& RELATED OBJECTS

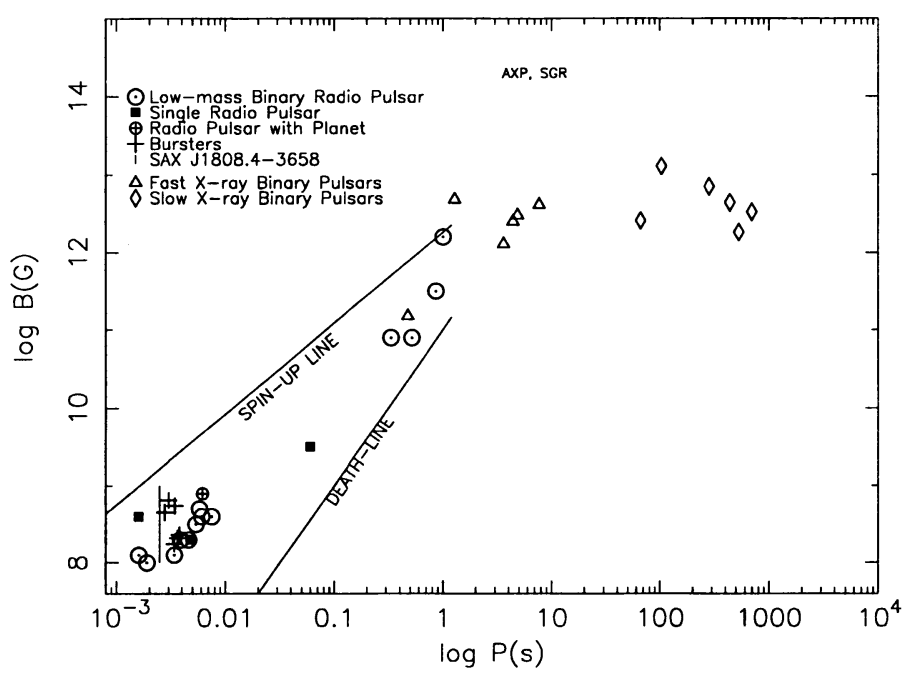

Figure 1. X-Ray Binary Pulsars and Related Objects. Accreting pulsars are shown for which magnetic fields are estimated, from cyclotron features for periods longer than $1 \mathrm{~s}$ and on the basis of assumed equilibrium for periods shorter than $10 \mathrm{~ms}$ (White \& Zhang 1997). Radio pulsars expected to have come from low-mass, binary X-ray pulsars are shown. The tentative B-P region of "Anomalous X-ray Pulsars" (AXP) and "Soft Gamma Repeaters" (SGR) is labeled.

Cen X-3 with its $4.85 \mathrm{~s}$ period appeared to exhibit long term speed-up at about the rate that the observed accretion would imply if the magnetic field at the surface were about $10^{12}$ Gauss and if this was close to the fields of young pulsars like the Crab pulsar, as obtained from their spin-down rates. The first detection of a spectral feature that could be associated with transitions in a strong magnetic field was twenty years ago (Trümper et al. 1978). The Her X-1 flux was falling fast with energy at the energy of the feature, and it was uncertain whether absorption or emission was being seen. By now, the significance of the feature and the ability to measure it at different phases of the pulse period have established that it is usually in absorption. A few other sources exhibited cyclotron resonance features; in particular, during a transient outburst of $3 \mathrm{U} 0115+63$, the first evidence of a fundamental and first harmonic appeared. Ginga detected a feature in enough sources to suggest a connection between the continuum spectrum and the cyclotron resonance feature (Mihara, Makishima, \& Nagase 1998). In 1996, BeppoSAX and RXTE both fielded instruments with special sensitivity to cyclotron lines in the energy range $10-100 \mathrm{keV}$.

The number of detections has risen, and some systematic correlations are emerging which are beginning to test theories of the emission region. The results for the cyclotron absorption energies and the assumed dipole magnetic field are summarized in the table. 
Table 1. Cyclotron Lines and Magnetic Fields

\begin{tabular}{|c|c|c|c|c|c|c|c|}
\hline $\mathrm{P}(\mathrm{s})$ & Source ${ }^{1}$ & $\mathrm{E}_{1}^{2}$ & E2 & E3 & $\mathrm{E} 4$ & $\mathrm{~B}^{3}$ & Mission $^{4}$ \\
\hline 1.28 & Her X-1 & 42.1 & & & & 4.8 & Balloon,H,G,X,S \\
\hline 3.61 & $(\mathrm{~T}) 4 \mathrm{U} 0115+63$ & 12.4 & 21.4 & 33.6 & 48.5 & 1.1 & $\mathrm{H}, \mathrm{G}, \mathrm{X}, \mathrm{S}$ \\
\hline 4.4 & (T) $\mathrm{V} 0331+53$ & 27.2 & & & & 2.5 & G \\
\hline 4.85 & Cen X-3 & 27.9 & & & & 3 & $\mathrm{~S}$ \\
\hline 7.7 & $4 \mathrm{U} 1626-67$ & 37 & & & & 4.1 & $S, X$ \\
\hline 66 & (T)Cep X-4 & 28.8 & & & & 1.2 & $\mathrm{G}$ \\
\hline 104 & (T)A $0535+262$ & 55 & 115 & & & 13 & $\mathrm{C}$ \\
\hline 283 & Vela $\mathrm{X}-1$ & 24.5 & 54.4 & & & 7.0 & G, S, X \\
\hline 438 & $4 \mathrm{U} 1907+09$ & 18.9 & 39.4 & & & 4.4 & $\mathrm{G}, \mathrm{S}$ \\
\hline 529 & 4U 1538-52 & 20.6 & & & & 1.8 & G \\
\hline 696 & GX 301-2 & 37.6 & & & & 3.3 & G \\
\hline
\end{tabular}

$\overline{{ }^{1} \text { Names of transient sources are preceded by (T). }{ }^{2} \text { Energy in keV at centroid of feature. }{ }^{3} \text { Surface }}$ dipole field in $10^{12}$ Gauss. ${ }^{4}$ Missions carrying instruments which showed evidence for one or more cyclotron features in the spectra were, after the balloon flights, HEAO-1 (H), Ginga (G), CGRO (O), RXTE (X), and BeppoSAX (S). See Mihara et al. 1998; Dal Fiume et al. 1999; Heindl et al. 1999; and Santangelo et al. 1999.

Three of the sources in the table are transients and the absorption features; especially, the higher harmonics are observed when the source is bright, presumably because the increased accretion flow is necessary for sufficient optical depth in the absorption feature.

The observations of harmonics at about the correct spacing confirms expectations about the energy levels of electrons in the strong magnetic fields. While the agreement is to $10-15 \%$, the best fits do not confirm an exact integral ratio. However, the ratio is not expected to be exactly integral, and there are reasons for different harmonics to be formed at different heights. It seems plausible that the complexity of the accretion process could easily lead to sufficiently different locations for particular processes to dominate to explain the differences. When the data are good enough, the fits to spectral models require a high energy cutoff in addition to the resonance absorption.

The widths of the observed features are tens of $\mathrm{keV}(E / \Delta E \approx 2-4)$. The full-width-half-maxima appear to be proportional to the energy of the fundamental and consistent with Doppler broadening in thermal plasma with $\mathrm{kT}=$ 3-20 keV (Dal Fiume et al. 1999). The observations that have already taken place contain more detailed information about the behavior of the features as a function of spin phase and should soon significantly constrain detailed pictures of the flow of accreting gas onto the neutron star.

\subsection{Quasiperiodic Oscillations}

Under some circumstances, there are strong quasiperiodic oscillations (QPO) in the flux from accreting pulsars (Kommers, Chakrabarty, \& Lewin 1998; Heindl et al. 1999; Finger 1998). Two reports of detailed studies of QPO in RXTE data have been inconsistent with the magnetic beat precession model which was first thought likely to be the explanation. In these cases, the cyclotron line now provides independent knowledge of what the magnetic field is. With this knowl- 
edge, it appears that there is structure in the disk outside the magnetosphere, in structures in the disk. During the recent outburst of $3 \mathrm{U} 0115+63$, quasiperiodic oscillations appeared at $2 \mathrm{mHz}$ with a $5 \%$ rms amplitude. The $48 \mathrm{mHz}$ QPO in 4U 1626-67 also suggests blobs in Kepler motion outside the Alfvén shell.

While the very low frequency oscillations suggest origins outside the magnetosphere, evidence for characteristic time-scales of the polar cap or the magnetic field near the surface of the neutron star are sought at frequencies of $10-1000 \mathrm{~Hz}$. For an observation for a binary orbit of Cen X-3 when the source was very bright, Jernigan, Klein, \& Arons (2000) found a broad peak in summed power spectra which they find comparable to results of simulations of accretion onto a magnetic pole in which "photon bubble oscillations" are excited.

\subsection{Weak Magnetic Field Pulsars}

More than fifty X-ray sources, mostly in the galactic bulge, have faint optical counterparts and are presumed to be low-mass X-ray binaries which have been accreting for the lifetime of the low-mass companion, some $10^{8} \mathrm{yr}$. Since pulsations had not been found in deep observations by HEAO-1, Exosat, Ginga, and other missions even when time resolution could have detected periods as short as a few milliseconds, it has been clear that the neutron stars and accretion flow were different from those in the young, high-mass X-ray binaries. The weak fields of older radio pulsars have suggested that the old neutron stars in X-ray binaries have correspondingly low magnetic fields. Exactly how weak they are is still uncertain but probably not weak enough to completely explain why the $\mathrm{X}$-ray flux produced in accretion is not pulsed. Sufficient optical depth to scattering may be able to explain it, but, in that case, lower luminosity sources with lower accretion rates may have less obscured lighthouse beams.

Now we have one bona fide pulsing burster, XTE J1808-3658. In June 1998, RXTE's PCA field of view serendipitously scanned over a transient in the galactic bulge (Marshall 1998). It appears to be the same source as SAX J1808.43658 , discovered in an outburst 18 mo. before (in't Zand et al. 1998). Follow-up observations discovered (Wijands \& van der Klis 1998) that it had coherent pulses at a frequency of $401 \mathrm{~Hz}$. The observed period varied, as Chakrabarty \& Morgan proved (1998), with the Doppler shifts of a 2 hour binary. During the transient's first appearance, two bursts occurred with a fast rise and an exponential cooling decay that is the definition of Type I bursts that are believed to be thermonuclear flashes on a neutron star. This is in contrast to the "Bursting Pulsar" GRO J1744-28, which has a long pulse period (0.5 s), and to bursts that fit the mold of Type II bursts thought to be from an accretion instability.

Extensive PCA observations of the source traced its 25 day decay. At a distance argued to be $4 \mathrm{kpc}$ (from the peak luminosity of the bursts), it had a peak luminosity of $5 \times 10^{36} \mathrm{erg} \mathrm{s}^{-1}$ and was still pulsing in the last PCA observations at $4 \times 10^{34} \mathrm{erg} \mathrm{s}^{-1}$. Neither the spectrum nor the pulsed fraction appeared to change. From these facts were deduced a field of $5 \times 10^{8}$ Gauss, along with constraints on the mass-radius relation (Psaltis \& Chakrabarty 1998; Burderi \& King 1998).

Chakrabarty \& Morgan (1998) found a very small mass function. Although the signal is very strong, the value of $\sin (62.8 \mathrm{lt}-\mathrm{ms})$ would suggest an improbably small inclination angle unless the companion is less massive than a main sequence 
star filling its Roche lobe in a 2 hour orbit. If it turns out that we can only see the pulsations looking at the system face on, we may never find very many.

However, it is possible that the fields of brighter sources are weaker or the poles obscured, and that SAX J1808.4-3658 is the first to be found of a population of sources. It is clear from BeppoSAX Wide Field Camera results that there are in the galactic bulge many transient bursters which, as transient persistent sources, do not get brighter than about $100 \mathrm{mCrab}$. Some of those may also be fast pulsars. In view of that possibility, we have been making biweekly scans of the galactic center region. These PCA scans have detected new transients down to a level of a few milliCrab, some with very interesting variability (e.g., XTE J1819-25=V4631 Sgr); but so far no coherent pulsations have been found.

\section{Nonpulsing, Low-Mass X-ray Binaries}

The 50 low-mass $\mathrm{X}$-ray binaries divide into the luminous $\mathrm{Z}$ sources (only 6 sources), the luminous "atoll" sources ( 5 agreed upon), the less luminous and regularly bursting atoll sources (at least a dozen), and over 20 sources which are bursters but have weak persistent flux. Z and atoll sources are both capable of bursting, in that GX $3+1$ among the atolls and Cyg X-2 among the $\mathrm{Z}$ sources do sometimes burn unstably and exhibit bursts.

The $\mathrm{kHz}$ pulsations that RXTE discovered have appeared in persistent flux measurements in all of the $\mathrm{Z}$ sources, in none of the bright atoll sources, in all the atoll bursters, and in some of the faint bursters. Bursts have been observed from many bursters. So far, oscillations have been seen in bursts from 6 of them. The $\mathrm{kHz}$ frequencies seen in the persistent flux are not seen in the burst flux. Vice versa, the burst oscillations have not been seen in the persistent flux.

The QPO seen in the persistent flux from these sources change in frequency by large amounts (factor of 2), and none of the models assign the rotation of the neutron star to these QPO periods. The QPO during the bursts change by less than about $1 \%$ and, in the only detailed model, the period is the rotation of the neutron star. These oscillations strongly suggest that even the sources which do not have coherent high-frequency pulsations are fast rotating neutron stars.

\subsection{Burst Oscillations}

The sources from which bursts have exhibited oscillations remain $4 \mathrm{U} 1728$ $34(363 \mathrm{~Hz}), 4 \mathrm{U} 1636-53(581 \mathrm{~Hz}), 4 \mathrm{U} 1702-43(330 \mathrm{~Hz}), \operatorname{Aql~X}-1(549 \mathrm{~Hz})$, KS 1731-26 $(524 \mathrm{~Hz})$, and MXB $1743-29(589 \mathrm{~Hz})$. Not all bursts from these exhibit oscillations. Figure 2 shows spliced data segments containing 6 bursts in which 5 of the 6 exhibit the same asymptotic frequency and similar variations by a few $\mathrm{Hz}$ in the frequency as the burst progresses. It is not understood why they are sometimes not present. It remains plausible that the burst sources for which no burst oscillations have been found are still fast rotating neutron stars and may at some time exhibit oscillations. Exosat observations of bursters established that these sources have cycles of different burst characteristics.

There is evidence that, despite the small but definite change in frequency in a burst, that at least the oscillations during the cooling tails of bursts are coherent. Strohmayer \& Markwardt (1999) showed that, for a simple frequency 

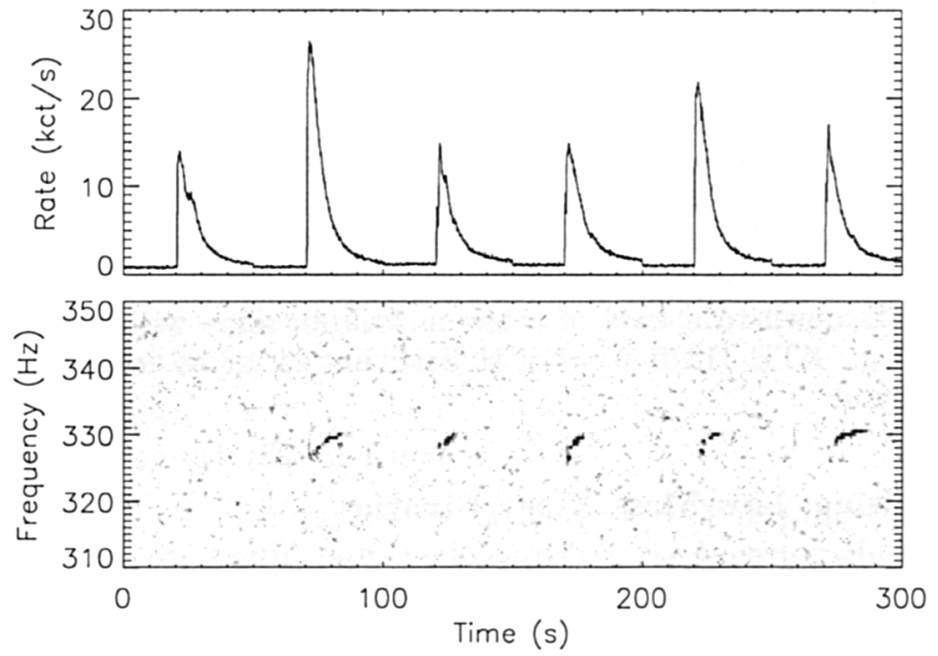

Figure 2. Burst Oscillations in An Atoll Burster (C. Markwardt). Sections of data containing bursts from $4 \mathrm{U}$ 1702-43 are adjoined in the top panel. In the bottom is a greyscale dynamical power spectrum showing the frequency range of the burst oscillations.

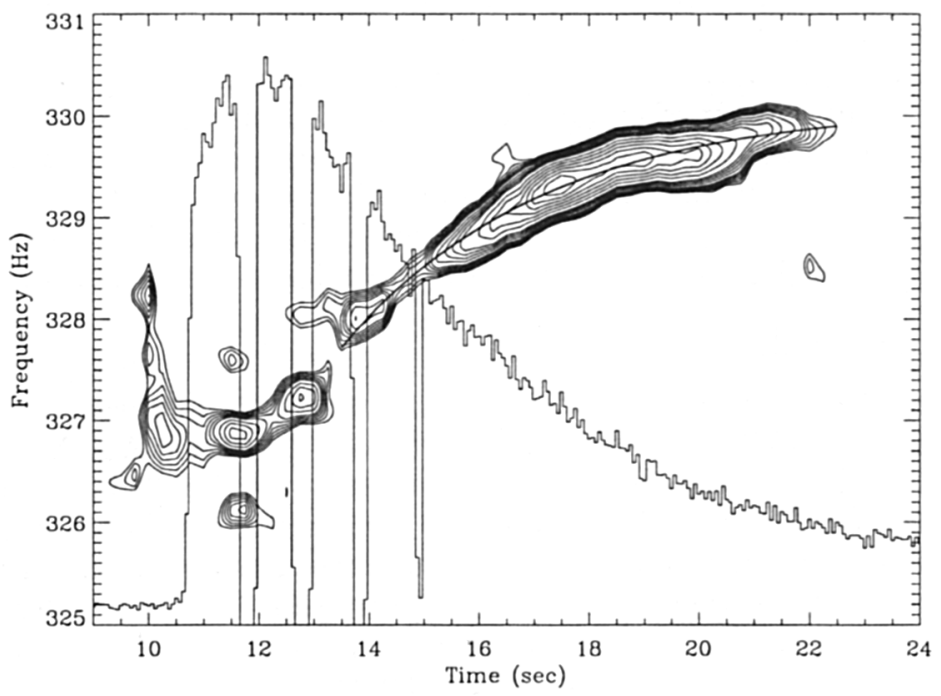

Figure 3. Variable Frequency of Oscillations during Burst (T. Strohmayer). The burst light curve is superposed. (Gaps occur where the data readout was not fast enough for this high time-resolution data.) 
function of time $\nu(t)$, the Fourier power is maximized with the phase of photons arriving at time $t_{\jmath}$, given by $\phi_{\jmath}=2 \pi \int_{0}^{t_{j}} \nu\left(t^{\prime}\right) d t^{\prime}$. Figure 3 shows contours of smoothed power in $2 \mathrm{~s}$ intervals for the second burst in Figure 2. The frequency function of time shown by the line is a good fit to the assumption of coherence during that interval.

In a number of bursts, the time dependence of the frequency suggested by the power spectra is more complex. A very strong signal during the initial rise of the burst can be interpreted in terms of 1 or 2 hot spots growing in size as the burning area increases to cover the whole star (Strohmayer, Zhang, \& Swank 1997). There is often a gap between that signal and the signal appearing in the burst tail. An asymmetry on the neutron star could lead to a single hot spot. If the star has an approximately dipole field, two poles might be expected to stand out as hotter than the surroundings, and the observed frequency could be the harmonic of the spin frequency. Miller (1999) found that for $4 \mathrm{U} 1636-53$ there was evidence for a subharmonic $(290 \mathrm{~Hz})$ of the dominant signal, briefly at the beginning of some bursts. Otherwise, the subharmonics and harmonics have amplitudes more than an order of magnitude less than the main frequencies.

\subsection{Persistent Flux QPO}

The typical kilohertz QPO signals in the persistent flux are 2 peaks, one narrower than the other, which move in frequency as the luminosity moves, with timescales of hours. One or both of the features may become broad and faint for a time. When the frequency wanders and both are present, they wander together. They keep approximately the same interval between them, although this interval appears to shrink by as much as $20 \%$ at the upper end of the ranges that have been observed.

The $\mathrm{Z}$ sources, the bright atoll sources, and the atoll burst sources all have substantial structure in the lower frequency part of power spectra, below $100 \mathrm{~Hz}$. This is where the QPO discovered with Exosat appeared, with their correlation with the luminosity. For each Z source (see Wijnands \& van der Klis 1997) when the source is on its "Horizontal Branch", both Horizontal Branch Oscillations (HBO) and kilohertz QPO are present, and their changes with luminosity are correlated. The HBO is different from the kilohertz QPOs in having a strong harmonic, while there has been no evidence for any harmonics or subharmonics for the kilohertz oscillations. It is characteristic of the power spectra on the horizontal branch of $\mathrm{Z}$ sources to have band-limited white noise at frequencies below a break frequency, with the HBO just above the break. All of these features are usually correlated with the luminosity on that branch.

Qualitatively, the atoll bursters have not only kilohertz oscillations similar to the more luminous $\mathrm{Z}$ sources but also similar low-frequency power spectra. They have band-limited white noise which was a defining characteristic of atoll sources, and, above the break, they have a strong additional QPO feature. Their $\mathrm{HBO}$ are broader with less clear harmonic structure and more variation in the low-frequency QPO. Ford \& van der Klis (1998) document that for 4U 1728-34 the break frequency and what they call the "lower frequency Lorentzian" are strongly correlated. The correlation $\nu_{L} \propto \nu_{u}^{2}$ is just the relation that LenseThirring precession would imply for clumps in Kepler motion in tilted circular orbits close to the innermost circular orbit (Stella \& Vietri 1998). 
Consequences of models of the kilohertz QPO have been under development as the observed characteristics are being explored. These very different models each involve some physics which appears relevant. Each also has some points of possible difficulty.

The sonic point model (Miller, Lamb, \& Psaltis 1998) provides a mechanism for the Kepler frequency and the rotation frequency of the neutron star to beat without assuming a strong magnetic field and without the dependence on luminosity that the magnetic beat frequency model implied. The sonic point determines the inner radius of the disk, where blobs circulate at the Kepler frequency. The spin beats with the Kepler blobs to produce the lower kilohertz frequency. But because the $\mathrm{HBO}$ are also observed and appear to be well described by the magnetic beat frequency model, it is currently supposed that the magnetosphere balances accretion only partially. The model assumes that the accreting neutron star does have poles that are hotter than the rest of the star, but the effect of only one of these is observed in the beats. The difference frequency between the two kilohertz frequencies is approximately the spin frequency. It can be reduced, however, because the radiation interacts with blobs that are spiraling into the neutron star.

Stella \& Vietri (1999) also pointed out that, for slightly elliptical orbits, the epicyclic motion would have a frequency which, if it beats with the Kepler motion, could produce the lower kilohertz frequency. While plausible eccentricity and neutron star mass could fit the data for the best measured case (Sco X-1), the magnitude of the Lense-Thirring precession would require a neutron star moment of inertia 2-4 times larger than that for any derived equation of state. No mechanism has been detailed for radiation variation at these frequencies.

The sometimes very narrow QPO have also suggested a resonant oscillation of some sort. In one model involving resonances (see Titarchuk, Osherovich, \& Kuznetsov 1999), the plasma in the boundary between the disk and magnetosphere undergoes radial and vertical oscillations as it circulates with the magnetosphere. In this case, the lower kilohertz frequency is identified with the Kepler frequency, the higher kilohertz frequency with a plasma frequency called "upper hybrid", and the HBO frequency with the low branch. Assumption that the inner disk interacts with the boundary of the neutron star rather than the magnetosphere can possibly explain other features in the power spectra.

\subsection{The Role of Accretion in the QPO}

A remarkable aspect of the kilohertz oscillations has been the fact that the phenomenon and the numerical range of frequencies are independent of the average luminosity of the sources. Thus, the frequencies range from $400 \mathrm{~Hz}$ to $1200 \mathrm{~Hz}$ for an atoll burster with peak luminosity of perhaps $10^{37} \mathrm{erg} \mathrm{s}^{-1}$, just as it does for a $\mathrm{Z}$ source with peak luminosity above $10^{38} \mathrm{erg} \mathrm{s}^{-1}$. This first suggested that the frequency must depend on something independent of the accretion rate, such as the innermost stable orbit or the radius of the neutron star (Zhang, Swank, \& Strohmayer 1997). However, for a given source, over short ranges of luminosity for periods of time less than or on the order of a day, the frequency increases with luminosity. Assuming that one of the frequencies is a Kepler frequency, it should saturate at the frequency of motion in the innermost stable orbit. Obser- 
vations of $4 \mathrm{U}$ 1820-30 have seemed to show this effect. (See recent confirmation by Kaaret et al. 1999.)

Another aspect of this independence of luminosity between sources is that a given source can go through the same frequency run for different ranges of luminosity. Mendez (1999) has put together the data from 4 sources that show this characteristic. On the other hand, the frequency varies systematically around the atoll in a color-color diagram. These characteristics suggest that for the atoll bursters there is a well-defined emission geometry and mechanisms which produce the QPO. The frequencies and spectra may be related to some mass flow rate in the system, but the luminosity is not uniquely related.

\subsection{Phase Lags}

The PCA has been used to measure the phase or time lags between different energy bands for many sources and for many of the temporal phenomena, the continuum noise in power spectra, various QPO in the persistent flux, and the oscillations in the bursts. Hard lags have been known, since Exosat and Ginga, to characterize black hole candidate noise and HBO. They also appear to characterize the LMXB noise (Ford \& van der Klis 1999). However, the lower of the two kilohertz frequencies is definitely subject to soft lags, although they are very small in comparison to the soft lags of SAX J1808.4-3658. Lags for the bursts and the upper kilohertz QPO are small, and the situation is not yet clear. While there are possibilities of either Compton up- or down-scattering, and Doppler shifts can cause lags, definitive patterns and consistent interpretations are uncertain.

\section{Conclusions}

The new results on accreting binary neutron stars found by RXTE and BeppoSAX provide new tools to gain physical insight both into the compact objects that are the last equilibrium point before collapse to a black hole and into the binary accretion flows onto them.

In the case of the strong field accreting pulsars, cyclotron features allow direct measurement of their magnetic fields, and the timing signatures of the flow then illuminate apparent irregularities in the disks. The conditions of their occurrence are not clear, and they are far from being well studied. There are hints of vibration signatures that might come from the accretion mound above the polar cap and are possible evidence for photon bubbles, but they need confirmation and more study.

Magnetic fields down near $10^{8}$ Gauss in low-mass X-ray binaries are confirmed by the discovery of one irrefutable example. The uncertainty of important parameters of the system leave it still ambiguous whether we should find more such systems. A burster itself, it could resolve many technical questions about the QPO in LMXB if it ever manifests them.

Evidence continues to support the conclusion that bursters are fast rotating neutron stars with low fields. The burst frequency and the difference between two high frequencies in the persistent flux seem rather clearly related, but the phenomena are still complex in detail.

The persistent flux QPO around $1 \mathrm{kHz}$ and around $100 \mathrm{~Hz}$ are correlated; 
and the correlations appear very general, holding for atoll sources, Z sources, and possibly black hole candidates, as well. Competitive models are still working out detailed implications for comparison. It is notable that they all involve effects within a few kilometers of the neutron star, where effects of General Relativity are involved.

\section{References}

Chakrabarty, D., \& Morgan, E. H. 1998, Nature, 394, 346

Dal Fiume, D., et al. 1999, Adv. Space Res., in press

Finger, M. H. 1998, Adv. Space Res., 22, 1007

Ford, E. C., \& van der Klis, M. 1998, ApJ, 506, L39

Ford, E. C., van der Klis, M., Mendez, M., van Paradijs, J., \& Kaaret, P. 1999, ApJ, 512, L31

Heindl, W. A., Coburn, W., Gruber, D. E., Pelling, M. R., Rothschild, R. E., Wilms, J., Pottschmitdt, K., \& Staubert, R. 1999, ApJ, 521, L49

in't Zand, J. J. M., Heise, J., Muller, J. M., Bazzano, A., Cocchi, M., Natallucci, L., \& Ubertini, P. 1998, A\&A, 331, L25

Jernigan, J. G., Klein, R. I., \& Arons, J. 2000, ApJ, in press

Kaaret, P., Piraino, S., Bloser, P. F., Ford, E. C., Grindlay, J. E., Santangelo, A., Smale, A. P., \& Zhang, W. 1999, ApJ, 520, L37

Kommers, J. M., Chakrabarty, D., \& Lewin, W. H. G. 1998, ApJ, 497, L33

Marshall, F. E. 1998, in IAU Circ. 6876, ed. D. W. E. Green

Mendez, M. 1999, in Proc. 19th Texas Symp. on Relativistic Astrophysics and Cosmology, eds. J. Paul, T. Montmerle, \& E. Aubourg (Paris: World Scientific Press), in press

Mihara, T., Makishima, K., \& Nagase, F. 1998, Adv. Space Res., 22, 987

Miller, M. C. 1999, ApJ, 515, L77

Miller, M. C., Lamb, F. K., \& Psaltis, D. 1998, ApJ, 508, 791

Santangelo, A., et al. 1999, ApJ, 523, L85

Stella, L., \& Vietri, M. 1998, ApJ, 492, L59

Stella, L., \& Vietri, M. 1999, Phys. Rev. Lett., 82, 17

Strohmayer, T. E., \& Markwardt, C. B. 1999, ApJ, 516, L81

Strohmayer, T. E., Zhang, W., \& Swank, J. H. 1997, ApJ, 487, L77

Titarchuk, L., Oscherovich, V., \& Kuzntzov, S. 1999, ApJ, 525, L129

Trümper, J., Pietsch, W., Reppin, C., Voges, W. Staubert, R., \& Kendziorra, E. 1978, ApJ, 219, L105

White, N. E., \& Zhang, W. 1997, ApJ, 490, 87

Wijnands, R., \& van der Klis, M. 1997, in Accretion Processes in Astrophysics: Some Like it Hot!, eds. S. S. Holt \& T. R. Kallman (New York: AIP), 381

Wijnands, R., \& van der Klis, M. 1998, Nature, 394, 344

Zhang, W., Swank, J. H., \& Strohmayer, T. E. 1997, ApJ, 482, L167 